Trabajos de: Prehistoria

48, 1991, PP. $365-372$

\title{
EL MOSAICO DE LAS ESTACIONES DE CORDOBA
}

\author{
POR
}

\author{
GUADALUPE LOPEZ MONTEAGUDO (*)
}

RESUMEN El trabajo tiene por objeto dar una lectura aproximada de tres fragmentos de mosaicos, conservados en el Museo Arqueológico de Córdoba, de confusa interpretación. La autora aporta nuevos datos para la posible identificación de las figuras que decoran los medallones circulares, a través del estudio iconográfico de las mismas y de su situación en las esquinas del pavimento, llegando a la conclusión de que se trata de una representación abstracta de las Estaciones que, en esta ocasión, son evocadas mediante personajes sacados de la mitología.

ABSTRACT It is the purpose of this research to provide for an approximate interpretation of three mosaic fragments, preserved in the Archaeological Museum of Córdoba. The author supplies new evidence in support of a possible identification of the figures decorating the circular medallions. In the light of the iconographic study of the figures and their location in the corners of the floor, the author reaches the conclusion that they are an abstract representation of the Four Seasons, which on this occasion are expressed through characters taken from mythology.

Palabras clave Musivaria. Mitología. Estaciones. Báquico. Sileno. Ganimedes. Europa. Constelaciones. Attis. Atributos. Adonis. Sarcófagos. Ierogamia. Hellotia. Toro. Flores. Vientos. Zeus.

Key words Mosaic art. Mythology. Seasons. Silenus. Ganymede. Europe. Constellations. Attis. Atributes. Adonis. Sarcophagus. Ierogamia. Hellotia. Bull. Flowers. Winds. Zeus.

En el Museo Arqueológico de Córdoba se conservan tres fragmentos de un mosaico, aparecidos en la zona que en la actualidad ocupa el Banco de España en la capital cordobesa. Se trata de tres medallones circulares, de $42 \mathrm{~cm}$. de diámetro, con empleo de teselas de pasta vítrea en verde y azules de diferentes tonalidades (Blázquez, 1981: 27, núm. 11, lám. 12) (1).

De los restos conservados se deduce que los tres fragmentos formaban parte de los ángulos de

(') Dpto. de Historia Antigua y Arqueologia. Centro de Estudios Históricos. CSIC. Duque de Medinaceli, 6. 28014 - Madrid.

(1) Deseo expresar mi agradecimiento más sincero a los Dres. Alejandro Marcos Pous y Ana María Vicent Zaragoza por la desinteresada ayuda que me han prestado en el estudio de estos fragmentos, facilitándome valiosos datos sobre los mismos, así como las fotos que ahora se publican. 
un pavimento con orla de flores cuatripétalas en negro sobre fondo blanco, determinando la intersección de los vértices de sus hojas círculos tangentes. Sigue una banda de ajedrezado, en blanco y negro, sobre la que se apoyan los medallones bordeados en sus tres cuartas partes por un sogueado de tres cabos en blanco, ocre y marrón y otra franja interior más estrecha, también de ajedrezado en blanco y negro, que completa el círculo en su totalidad. Los espacios triangulares resultantes de la intersección de los círculos con las líneas rectas que forman el ángulo se han rellenado de teselas de color negro.

El interior de los medallones va decorado con bustos que fueron interpretados, en un primer momento y creemos que acertadamente, por Blázquez como la representación de las Estaciones. La identificación de uno de ellos con el Otoño (lám. I, 1) no ofrece dudas, ya que se trata de una figura masculina barbada y coronada de pámpanos de color verde, que se cubre el hombro izquierdo con un manto de tonalidad ocre. Está vista en tres cuartos hacia la derecha y representa a un hombre de edad ya avanzada. Blázquez rectificó su primera interpretación del Otoño en un trabajo posterior, inclinándose por una representación de Sileno o la personificación de un río (Blázquez, 1982: 84). Un busto semejante de viejo barbado y coronado de pámpanos, al que Blanco cree poder identificar con Sileno, aparece en el mosaico de los bustos báquicos de Itálica (Blanco, 1978 b: 28, lám. 13). No existe ninguna contradicción entre ambas interpretaciones, ya que, dada la situación de este medallón en el ángulo del pavimento, es posible que la figura de Sileno, con su corona de hojas de vid, se haya utilizado como la personificación o la alegoría del Otoño.

Más problemas ofrecen los dos medallones siguientes, en uno de los cuales se representa un busto de difícil identificación (lám. I, 2). La figura está vista también de tres cuartos hacia la derecha, como sus compañeras, pero en esta ocasión la cabeza se vuelve hacia la izquierda. Lleva clámide o vestido de color verde que cubre el hombro izquierdo y se abrocha sobre el derecho, idéntico al de la figura siguiente. También los rasgos de ambas ofrecen ciertas similitudes, especialmente en el tratamiento de los labios. Fernández-Galiano se inclina a pensar que se trata de una representación de Ganimedes, basándose sobre todo en el tocado, una especie de bonete que recuerda el gorro frigio de este personaje (Fernández-Galiano, 1982: 19). Sin embargo, las reparaciones que afectan de forma notoria a esta zona del mosaico dificultan la identificación, aunque los rasgos afeminados de la figura, el tipo de indumentaria y el tratamiento de los cabellos recuerdan efectivamente al modelo iconográfico de Ganimedes. Su situación en la esquina del pavimento y la liviandad de su ropaje inducirían a identificarla con la representación del Verano. El mismo cabello, de color ocre oscuro y figurado de manera hirsuta, podría evocar elementos vegetales relacionados con la estación estival. No es extraña, por otra parte, la utilización de la figura de Ganimedes como símbolo o alegoría del Verano, ya que el mito de su rapto estaba originariamente conectado con el ciclo de la vegetación (Sichtermann, 1953: 13-17). La misma asociación puede conjeturarse para el águila que, tras la aventura del rapto, fue convertida en constelación, igual que lo fue el toro después de desempeñar su papel en el mito de Europa. Y precisamente en el calendario romano la constelación del águila aparece en el cielo a principios del mes de junio, vinculándose de esta forma con el período estival.

La ausencia, sin embargo, de algún atributo definitorio de la identidad de este personaje produce una cierta confusión, ya que la imagen de un joven frigio, como alegoría de un período estacional, tanto puede hacer referencia a Ganimedes, como a Attis o a Adonis. Attis se utiliza como alegoría de la Primavera en un sarcófago de Roma, por su carácter de dios de la resurrección (Hanfmann, 1951: II, 178, n 489), celebrándose la fiesta fúnebre para honrar su memoria en el equinocio de la Primavera, aunque es más frecuente su asociación con el invierno, como ocurre con la imagen de Adonis. Al parecer, el ancestro de Attis-Adonis como alegoría del Invierno es Ganimedes, el copero de los dioses que fue trasplantado al firmamento como signo de Aquarius, y al que se le representa en los calendarios para evocar los meses de enero y febrero como joven frigio sosteniendo una jarra (Hanfmann, 1951: I, 240).

El tercer busto (lám. II, 1) representa una figura femenina de tres cuartos hacia la derecha, con la vista dirigida hacia arriba. Viste traje de color verde, que cubre el hombro izquierdo y se abrocha 
sobre el derecho y peina el cabello en grandes ondas a ambos lados de la cabeza. Tras su hombro derecho asoma una cabeza de toro, de color ocre oscuro y cornamenta negra, identificada como tal por Fernández-Galiano, lo que le lleva a interpretar este grupo como una representación, un poco forzada por el espacio disponible, del mito de Europa (Fernández-Galiano, 1982: 19). La identificación nos parece perfectamente aceptable, pero con el añadido de que en este caso la figura de Europa se ha utilizado como alegoría de la Primavera (López Monteagudo y San Nicolás Pedraz, 1991: en prensa).

Varios argumentos pueden argüirse en favor de esta ecuación Europa-Primavera, que a continuación pasamos a exponer. En primer lugar, Europa forma parte de una de las divinidades fenicias trasplantadas al mundo helénico (Herod. IV 147 y VII 91; Paus. V 25,7; Serv. ad Aen. III 88), viendo Overveck y Jahn en la Europa cretense a una divinidad telúrica, cuya ierogamia con Zeus bajo el plátano de Gortyna, que en memoria de estos amores obtuvo el privilegio de no perder nunca sus hojas, era recordada cada primavera en la fiesta de las Hellotia (Athen. XV 678 a; Hesych. s.v.; Steph. Byz. s.v.). Esta fiesta tenía un carácter de resurrección y de apoteosis, ya que la evocación de la unión de Europa y Zeus contenía la idea de una teogamia mediante la cual el dios del cielo se mezclaba cada primavera con la tierra, penetrándola de un vigor nuevo (Hild, 1892: 863-864). La fiesta de las Hellotia coincidía con la aparición en el cielo de la constelación del toro hacia el 14 de mayo (Ovid. Fast. V 603-604), es decir el toro, cuya forma había adoptado Zeus para seducir a Europa, y que por este motivo fue convertido en constelación e incluido entre los signos del Zodíaco. Y precisamente el mito de Europa se introduce en los Fastos de Ovidio a propósito de este fenómeno astrológico (Wattel-de-Croizant, 1982: 84-85).

Existe, pues, una estrecha conexión entre el mito de Europa y la estación primaveral, por la simbología que la ierogamia entre la princesa sidonia y Zeus tiene en relación con la energía renovadora que la tierra recibe del cielo en primavera. Los detalles del mito también sugieren esta asociación: Europa aparece, junto a sus compañeras, recogiendo flores en un cesto en las playas de Tyro o de Sidon, tal como la describe Moschos (II 34, 37-62) y se la representa en una pintura de Stabiae, fechada en la mitad del siglo I d. C. (Schefold, 1972: 165-166, pl. XXX), o en moneda de Tyro, de mediados del III d. C. (Robertson, 1988: $\mathrm{n}^{0}$ 20). El cesto lleno de flores se documenta asimismo en un vaso pintado de Britania, datado en el siglo III d. C. (Toynbee, 1964: 417, pl. XCV a y b), y en el mosaico tunecino de Djemila, del siglo IV d. C., que representa ya el viaje marino con Europa sentada sobre el toro, el cual vuelve la cabeza hacia el cesto de flores que le ofrece la joven (De Croizant, 1972: 48-50, Fig. 10). Europa y sus compañeras adornan al toro con guirnaldas de flores en los mosaicos de Ouled Agla (De Croizant, 1972: 38-43, Fig. 7 b) y de Somerdale (Toynbee, 1964: 240-241, pl. LVII b), ambos fechados en el siglo IV d. C. Durante el viaje marino la princesa sidonia lleva en su mano una flor o una guirnalda, evocando los versos de Ovidio (Met. II 869-871) y de Moschos (II 30-99) que hacen referencia a la ocupación de la joven en el momento del rapto. Los documentos arqueológicos ofrecen algunas variantes iconográficas a este respecto, y así unas veces es una flor la que lleva Europa en la lucerna de Bulla Regia, datada en 150-200 d. C. (De Croizant, 1972: 34, Fig. 3), o un ramo de flores en el perdido mosaico de Naix-aux-Forges, del siglo II-III d. C. (Stern, 1960: no 243 B, pl. XLIII); una guirnalda sostiene la princesa sidonia en la terracota hispana de Sisapo, fechada en la primera mitad del siglo I d. C. (Fernández-Ochoa y San Nicolás, 1991: en prensa), y en el mosaico de Oudna, de mediados del siglo III d. C. (Besrour ben Mansour, 1979: 204 211): o también una corona de flores, tal como aparece en el camafeo del Cabinet des Médailles, de París, fechado en el siglo I-II d. C., y en sigillata de fines del II d. C. (Robertson, 1988: $\mathrm{n}^{\text {9 }} 188$ y 191). Europa sentada sobre el toro y llevando flores en su falda, a la manera de Proserpina, se representa en un relieve itálico en mármol, del siglo I-II d. C. (Robertson, 1988: $\mathrm{n}^{0}$ 171). La pradera florida está presente en el mosaico de Cannes, fechado en el siglo I d. C. (Wattel-de-Croizant, 1982: 91, pl. II), y en los pavimentos hispanos de Mérida, de mediados del II d. C. (Blanco, 1978a: 28, nº 4, lám. 5) y de Fernán Núñez, que se data en época severiana (Blázquez, 1981: 50-54, n 32, lám. 39). Esta presencia reiterativa de las flores sirven, a nuestro juicio, no sólo para evocar la ocupación de la princesa en 
el momento del rapto, sino como símbolo también de la estación en la que éste tiene lugar: la Primavera.

Así pues, varios detalles del mito, como es la insistente evocación de las flores por parte de los poetas y también la ierogamia de la princesa con Zeus a mediados del mes de mayo, coincidiendo con la aparición en el cielo de la constelación del toro, tienen un elevado contenido simbólico en relación con la primavera y así han sabido reflejarlo los documentos arqueológicos, algunos de los cuales hemos citado aquí a modo de ejemplos. No es extraño, pues, que en el medallón de Córdoba se haya utilizado la figura de Europa, teniendo como atributo al toro, como alegoría de la Primavera, ya que en esta representación convergen dos realidades: por un lado, el mito de Europa y, por otro, el tratamiento iconográfico de las Estaciones. Europa se ha representado a la manera tradicional de éstas: en forma de busto, dentro de un medallón que ocupa uno de los ángulos del mosaico, y con un atributo relacionado con el período estacional.

No sería, para concluir, el único ejemplo de esta asimilación, ya que la citada figura de joven recogiendo flores en un cesto, que decora una pintura de Stabiae conservada en el Museo Nacional de Nápoles, se interpreta indistintamente como la representación de Europa (Schefold, 1972: 165166, pl. XXX) y como la alegoría de la Primavera (Maiuri, 1953: 83). Por otra parte, la imagen del toro, que como signo astronómico aparece en el calendario romano a mediados del mes de mayo, se ha utilizado en diversas ocasiones como símbolo de la estación primaveral, por ejemplo en los mosaicos romanos de Saint-Romain-en-Gal, datado en el primer cuarto del siglo III d. C. (Lancha, 1981: 217-225, no 368), y de Dair Solaib, de la segunda mitad del V d. C. (Hanfmann, 1951: II, 154, no 192, Fig. 121), y también de los meses de abril y mayo, como ocurre en los mosaicos hispanos de Hellín (Blázquez et alii, 1989: 49-56, no 39, lám. 37) y de Fraga (Fernández-Galiano, 1986: 179-180), datados, respectivamente, en la primera mitad del siglo III y en la segunda mitad del IV d. C. La misma asociación aparece en un sarcófago romano de la segunda mitad del siglo III d. C., en donde la representación de la Primavera, como una joven con cesto de flores, va acompañada de la figura de un toro (Hanfmann, 1951: II, 187, A-6).

Falta el cuarto medallón, cuyo interior es de suponer que iría decorado con otra alegoría de las Estaciones.

Fernández-Galiano piensa que estos fragmentos cordobeses serían un ejemplo más, junto a los mosaicos de Itálica y de Fernán Núñez, de la representación de los Amores de Júpiter (FernándezGaliano, 1982: 19). Sin descartar esta idea, creemos que su identificación con las Estaciones parece tener, según hemos intentado demostrar, varias razones de peso: la situación de los medallones ocupando los ángulos del pavimento, lugar que se reserva normalmente para colocar a las personificaciones de las Estaciones o de los Vientos; el tipo iconográfico en forma de busto, siendo ésta la manera más frecuente de representar a las Estaciones (Abad, 1990: 17-23); los atributos alegóricos; y su mismo carácter simbólico. Que en este mosaico se han utilizado personajes o figuras mitológicas sacadas del Thiasos báquico o del ciclo de los Amores de Júpiter, es una posibilidad a tener en cuenta por la asociación de Baco con las Estaciones (Parrish, 1984: 43-45) y porque, a lo largo del siglo II d. C., las Estaciones aparecen conectadas a Zeus como responsable del ciclo del tiempo (Abad, 1990: 12). Tal vez tampoco sean ajenas a la concepción abstracta de estas representaciones mitológicas las corrientes filosóficas de la época.

Lo que sí es evidente es que no se trata de una representación convencional de las Estaciones, pero tampoco lo es su esquema compositivo que parece exclusivo de un taller hispano, y concretamente de la Bética, ya que sólo se documenta en otros dos mosaicos procedentes también de la provincia de Córdoba. El mosaico cordobés ofrece un esquema compositivo idéntico al del Thiasos báquico de Alcolea, datado en la segunda mitad del siglo II d. C., sólo que aquí se han colocado los bustos de los Vientos en los medallones circulares de las esquinas (Blázquez, 1981: 40-43, no 21, láms. 25 y 85). La misma composición se documenta en otro pavimento cordobés también báquico, de fines del siglo II d. C., cuyos ángulos van ocupados por cuatro rondos con las figuras de las Estaciones (Blázquez, 1981: 29-33, nº 12, láms. 13-15).

El esquema radial en torno a un octógono central (Salies, 1974: 12-14, 56-57; Bild 3, 43) se

T. P., 1991, $\mathrm{n}^{\mathrm{o}} 48$ 
atestigua en otros pavimentos hispanos, como el del Nacimieno de Venus, de Cártama, de finales del siglo II d. C. (Blázquez, 1981: 85-88, no 61, lám. 70), o el de la Medusa, de Mérida, de la misma fecha o de comienzos del siglo III d. C. (Blanco, 1978a: 49, no 57, lám. 88 B), pero en ellos faltan los círculos de los ángulos y los medios círculos en el centro de los lados, que caracterizan a los tres mosaicos cordobeses.

Fuera de Hispania el esquema que más se asemeja, aunque la decoración sea exclusivamente geométrica, es el de un pavimento de Djebel Oust, datado ya en el primer cuarto del siglo VI d. C. (Fendri, 1965: 167, Fig. 13). Un paralelo muy próximo a la composición cordobesa se encuentra en el mosaico con escena erótica de Piazza Armerina, del siglo IV d. C., también con círculos en los ángulos conteniendo cabezas femeninas y los bustos de las Estaciones en los hexágonos intermedios (Carandini et alii, 1982: I, 243-248; II, foglio XXXVI, 75).

Los fragmentos que nos ocupan debían formar parte de un mosaico de esquema radial en torno a un octógono central, al igual que los dos paralelos cordobeses citados, y como ellos tendría un contenido de carácter báquico. Así parece deducirse de la decoración que se conserva en uno de los espacios rectangulares yuxtapuesto al medallón con la posible alegoría del Verano, en donde se ven las patas anteriores de un cáprido, aunque también podría tratarse de un bóvido, y hacer alusión a las ideas representadas por los bustos de las Estaciones o a los mismos Amores de Zeus.

El pavimento se fecha, según Blázquez, a fines del siglo II d. C. o a comienzos del siguiente.

\section{BIBLIOGRAFIA}

Abad Casal, L. (1990): "Iconografía cie las Estaciones en la musivaria romana". Mosaicos Romanos. Estudios sobre Iconografia. Actas del Homenaje in Memoriam de Alberto Balil Illana (Guadalajara, 1990): 11-28. Guadalajara.

Besrour ben Mansour, S. (1979): «La mosaïque d'Europe de la Maison des Laberii». Antiquités Africaines XIV: 197-211.

Blanco Freijeiro, A. (1978a): Mosaicos Romanos de Mérida Corpus de Mosaicos Romanos de España I, Consejo Superior de Investigaciones Científicas. Madrid.

- (1978b): Mosaicos Romanos de Itálica (I). Corpus de Mosaicos Romanos de España II, Consejo Superior de Investigaciones Científicas. Madrid.

Blázquez, J. M. (1981): Mosaicos Romanos de Córdoba, Jaén y Málaga. Corpus de Mosaicos Romanos de España III, Consejo Superior de Investigaciones Científicas. Madrid.

- (1982): Mosaicos Romanos de Sevilla, Granada, Cádiz y Murcia. Corpus de Mosaicos Romanos de España IV, Consejo Superior de Investigaciones Científicas. Madrid.

Blázouez J. M. et alii (1989): Mosaicos Romanos de Lérida y Albacete. Corpus de Mosaicos Romanos de España VIII, Consejo Superior de Investigaciones Científicas. Madrid.

Carandini, A. et alii (1982): Filosofiana. La villa di Piazza Armerina. S. F. Flaccovio. Palermo.

DE Croizant, O. (1972): «Les représentations d'Europe en Afrique». Bulletin Archéologique du Comité des Travaux Historiques et Scientifiques VIII: 33-51.

FENDRI, M. (1965): «Evolution chronologique et stylistique d'un ensemble de mosaïques dans une station thermale à Djebel Oust (Tunisie)». La Mosaïque Gréco-Romaine I (París, 1963): 157-173.

FERNÁNDEZ-Galiano, D. (1982): «Nuevas interpretaciones iconográficas sobre mosaicos hispanorromanos». Museos $1: 17-27$.

- (1986): "El Calendario romano de Fraga». Boletín del Seminario de Estudios de Arte y Arqueología de la Universidad de Valladolid 52: 163-204.

Fernández-OchoA, C. y SAN Nicolás, P. (1991): "Terracotas romanas de Sisapo (La Bienvenida, Ciudad Real)». Homenaje al Profesor J. M. Blázquez: en prensa. Universidad Complutense. Madrid.

Hanfmann, G. M. A. (1951): The Season Sarcophagus in Dumbarton Oaks. Dumbarton Oaks Studies II, Harvard University Press. Cambridge.

Hild, J. A. (1892): "Europe». en Ch. Daremberg et Edm. Saglio (eds.): Dictionnaire des Antiquités Grecques et Romaines. Hachette, París: II/1, 862-865.

LANCHA, J. (1981): Province Narbonnaise. 2. Vienne. Recueil Général des Mosaïques de la Gaule. III/1. X supplément à "Gallia», Centre Nationale de la Recherche Scientifique. París.

López Monteagudo, G. y San Nicolás Pedraz, P. (1991): "La iconografía del rapto de Europa en el Mediterráneo 
occidental. A propósito de una lucerna del Museo de Sassari». Atti del VIII convegno di studio su "L'Africa romano, (Cagliari, 1990): en prensa, Sassari.

MaIURI, A. (1953): La peinture romaine. Editions d'Art Albert Skira, Genève.

PARRISH, D. (1984): Season Mosaics of Roman North Africa. Giorgio Bretschneider. Roma.

ROBERTSON, M. (1988): "Europa I». En Lexicon Iconographicum Mythologiae Classicae (LIMC), Artemis Verlag, Zürich-München: IV/1, 76-92, IV/2, 32-48.

SALIES, G. (1974): «Untersuchungen zu den geometrischen Gliederungsschemata römischer Mosaiken». Bonner Jahrbücher 174: 1-178.

SCHEFOLD, K. (1972): La peinture pompeianne. Essai sur l'évolution de sa signification. Traduction de J. M. Croisille, Collection Latomus 108. Bruxelles.

Sichtermann, H. (1953): Ganymed. Mythos und Gestalt in der antiken Kunst. Verlag Gebr. Mann. Berlín.

STERN, H. (1960): Province de Belgique. 2. Partie Est. Recueil Général des Mosaiques de la Gaule. 1/2. X supplément à «Gallia», Centre National de la Recherche Scientifique. París.

TOYNBEE, J. M. C. (1964): Art in Britain under the Romans. Clarendon Press. Oxford.

WATtEl-De-Croizant, O. (1982): «Ovide et l'Enlèvement d'Europe, aspects littéraires et mosalques du $1^{\text {er }}$ siècle». Caesarodunum XVII bis: 79-100. 

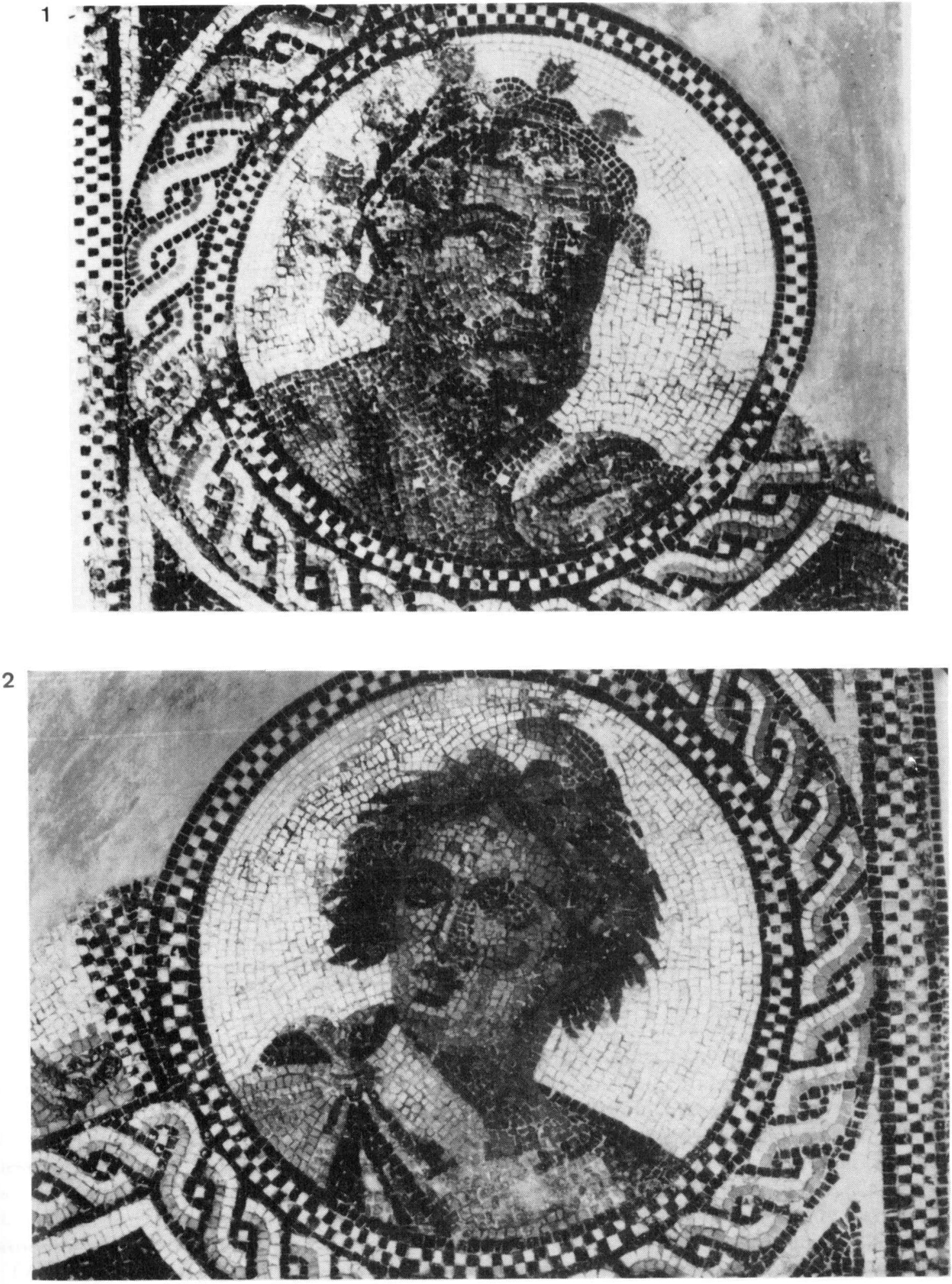

1. Busto del Otoño. (Foto cortesía de Ana M. ${ }^{\text {a }}$ Vicent de Marcos.); 2. Busto del Verano (?) (Foto cortesía de Ana M. ${ }^{a}$ Vicent de Marcos.) 


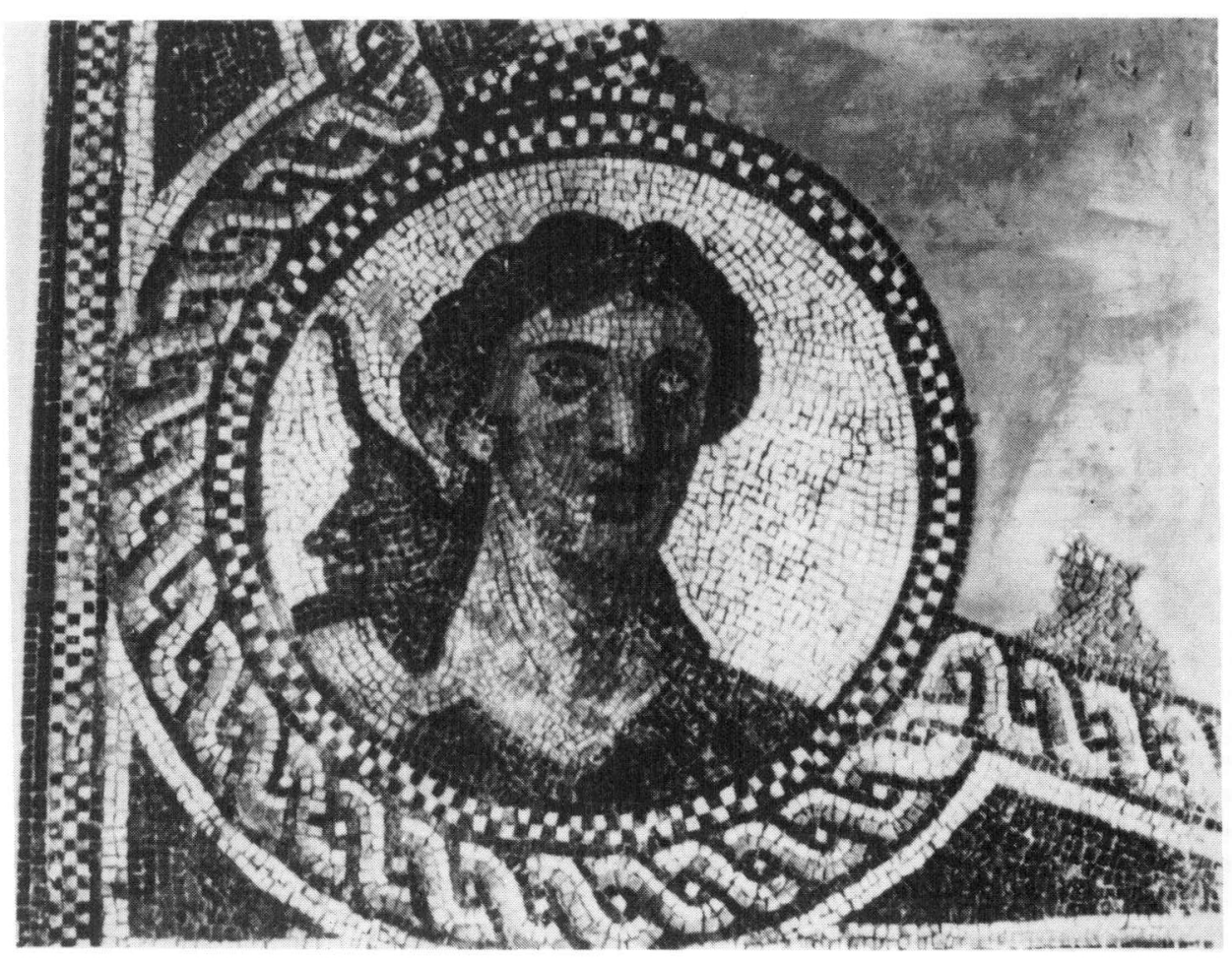

Busto de la Primavera. (Foto cortesía de Ana M. ${ }^{\mathrm{a}}$ Vicent de Marcos.) 